\title{
Overview of new PhDs in the Nordic Countries
}

Title: $\quad$ Economic Evaluation of a case management intervention for patients with chronic obstructive Pulmonary disease. How can patient heterogeneity be taken into account when evaluating the cost-effectiveness of a complex intervention entailing variation in service provision?”

Candidate: Sabrina Storgaard Sørensen

University: Department of Business and Management, Ålborg University, Denmark.

\begin{abstract}
:
In Denmark, the healthcare system is challenged as it is faced with an ageing population and an increasing number of patients diagnosed with chronic conditions. The management of chronic conditions is complex and requires a continuous effort from the individual patient and the healthcare system to prevent worsening of the condition. This includes various forms of care services across a range of health care providers and settings. For the healthcare system, chronic conditions lead to substantial economic challenges, which are only expected to be intensified in the coming decades. As a result, the Danish healthcare system has had a special focus on improved care strategies for chronic conditions in recent years. An example of one such strategy was the implementation of case management in the Danish regions and municipalities from the beginning of 2008.
\end{abstract}

The dissertation presents the economic evaluation of a case management intervention for patients suffering from chronic obstructive pulmonary disease (COPD). The case management intervention was defined as a complex intervention, as it consisted of a variety of care components tailored for the individual patient in accordance with patient needs and preferences. A randomised controlled trial was designed and conducted to assess cost-effectiveness, in which 150 patients with COPD were included. A cost-utility analysis demonstrated that the case management intervention was not deemed cost-effective. However, the study suffered from limitations which might have affected the results of the economic evaluation.

As studies evaluating complex interventions often include heterogeneous target groups, it may be appropriate to examine whether there exist subgroups for which the intervention would be cost-effective. However, currently there is no established methodology for the assessment of patient heterogeneity within economic evaluation, and the conventional methods for subgroup analysis are problematic in the evaluation of complex interventions containing both patient heterogeneity and service variation. The dissertation demonstrates an alternative method for assessing patient heterogeneity in the evaluation of complex interventions using latent class analysis (LCA) - a method which, until now, has been unexplored within health economic evaluation. LCA was conducted on registered process measures covering the variation on provided case management services at patient level in the intervention group. The method demonstrated three different patterns of service provision, and patient characteristics and cost-effectiveness varied across the identified patterns. The use of LCA revealed that it might be cost-effective to provide comprehensive case management, focusing particularly on the disease-specific components, for a subgroup of substantially diseased COPD patients.

LCA may enable more appropriate evaluation of complex interventions, which ultimately may lead to health gains for the patients and more efficient use of society's scarce resources. 
However, further research is needed to support the method's applicability for informing healthcare decision making.

Title: Hospital choice in times of restructuring the hospital sector

Candidate: Nasrin Tayyari Dehbarez

University: Department of Public Health, Aarhus University, Denmark. DEFACTUM, Central Denmark Region.

\section{Abstract:}

Hospital choice policy has been introduced in Northern European health care system, which aimed at increasing efficiency, enhancing providers' responsiveness and giving patients more power over the treatment.

Applying a health economics perspective, this $\mathrm{PhD}$ project investigated uptake of choice and the consequences of hospital choice policy on equity. The project investigated women's decision-making in relation to choice of birthing hospital, information seeking process and priorities regarding hospital characteristics. Furthermore, the utility of hospital attributes were quantified and trade-offs of hospital attributes were estimated.

To study uptake of choice and the effect of choice on equity of access, several Danish data registries were used. The study was a retrospective cohort of 134,049 women who were living near a non-highly specialized hospital and the bypassing of nearest hospital to reach a highlyspecialized hospital was assessed using multivariate logistic regression.

To study women's decision-making strategies and priorities regarding birthing hospital, thirteen women who were offered real choice of birthing hospitals at the first prenatal visit to the GP, were interviewed using a semi-structured interview guide and data were analyzed using a thematic analysis.

Moreover, to quantify women's preferences for hospital attributes, a discrete choice experiment with 12 choice scenarios was used. 517 women who were members of an online panel responded to choice sets by choosing between three unlabeled alternatives characterized by five attributes. A random parameter logit model was used to estimate the utility and marginal willingness to travel for improvements in other hospital attributes.

The results showed that $12 \%$ of women bypassed the nearest non-highly specialized hospital to reach a highly-specialized hospital. Notably, high education level was significantly associated with up-specialization.

The qualitative study informed us that women make decision independently and trust their own or peers' experiences when making decision. In addition, specialized services and qualified staff, continuity of midwifery care, hospital service offered and travel time were identified as the most important attributes of birthing hospital.

The results of discrete choice experiment showed that the key driver of choice of birthing hospital is availability of an neonatal intensive care unit; the study also revealed the relative importance of the hospital attributes. Substantial heterogeneity was observed due to prior experience with giving birth and with regard to risk attitude and health literacy.

This dissertation concludes that women's high demand for highly-specialized hospitals may reduce accessibility for those in need of specialized care and thereby threaten both equity and efficiency. In addition, women's demand for hospital services is mainly steered by previous birth experience and women's risk attitude. Therefore, there appears to be room for more information to be provided about the women's risk profile and service attributes as an instrument for making an informed decision. 
Title: $\quad$ Exploring time preferences and preventive behavior. The case of attendance to the gym

Candidate: Eskild Klausen Fredslund

University: Department of Health Services Research, University of Southern Denmark, Denmark

\begin{abstract}
:
Introduction: In this thesis I focus on theories from behavioral economics that may explain why some individuals do not achieve and sustain an optimal exercise level.

Data: In four papers I analyze the relation between time preferences and actual behavior as well as a break in exercise routines. Data used in the study was collected in collaboration with fitness $d k$. Subjects consisted of respondents to a questionnaire designed to elicit time preferences and attitudes towards working out, which was sent to members of fitness dk. Via the membership number I was able to connect members to actual exercise data from the registries of fitness $\mathrm{dk}$.

Results: In the first paper I analyze differences between time preferences measured in the monetary domain and the health domain. If time preferences for health and money are not the same, this can have consequences for the reliability of the body of literature using time preferences measured in the monetary domain to explain health related behavior.Assuming quasi-hyperbolic discounting including both present bias and long term discounting I find that time preferences measured in the monetary and health domain are remarkably similar.

In the second paper I analyze whether present biasedness and long term discounting as described by quasi-hyperbolic discounting can explain real exercise behavior in a gym. I find that a higher long-term discount rate is associated with lower gym attendance and that present bias is only significantly negatively correlated with gym attendance among individuals who do not face other issues, i.e. individuals who otherwise perceive gym attendance to be associated with low barriers and/or high benefits.

In the third study I analyze the decision to cancel ones membership and quit the gym. In a sample of newly enrolled members I find that more present biased individuals cancel their membership later. This leaves the present biased individuals facing a double whammy: they have lower attendance at the gym, and at the same time it is difficult for them to execute a cancellation of the membership.

In the fourth study I analyze the relation between behavioral routines and gym attendance. Using a regression discontinuity design I analyze whether the Easter Break introduces a discontinuous drop in exercise frequency. I find that there is a significant and relevant drop in exercise frequency following the Easter Break.

Conclusion: The thesis highlights the challenges that members of a fitness center may face in relation to achieving a sufficient and steady exercise frequency. Present biasedness and exercise routines appear to be key explanatory factors. This realization is relevant for citizens as well as policy makers concerned with introducing policies to improve the public's health through primary prevention.
\end{abstract}

Title: $\quad$ Economic Evaluation of Non-Pharmacological Interventions for Dementia: Methodology and Application of Decision Modelling

Candidate: Elizaveta Sopina

University: Danish Centre for Health Economics (DaCHE), University of Southern Denmark, Denmark

\footnotetext{
Abstract:

Dementia is an umbrella term for a variety of neurodegenerative diseases characterised by irreversible decline and loss of cognitive function, as well as a number of behavioural and
} 
functional problems. Currently available pharmaceutical treatments are supportive or palliative, rather than curative or disease-modifying, and, in large, focused on cognitive aspects of dementia, overlooking behavioural and functional outcomes. Real world studies have shown that these treatments are modestly effective at best, and have significant adverse side-effects. These limitations have led to an internationally-identified need for a broader approach to preventing, identifying and treating dementia through non-pharmaceutical interventions (NPIs) which target a wide spectrum of dementia symptoms: cognitive, psychological/behavioural, and functional aspects, among others. However, due to broader effects of NPIs, economic evaluation, and in particular, decision modelling, requires a more specialised approach.

This project focused on investigating decision modelling NPIs in dementia, and consisted of six papers/studies. First, a systematic review of economic decision models for NPIs in dementia was conducted and found that application of pharmaceutical models to NPIs in not suitable due to differences in outcome measure and time-horizons, and purposefully-developed models should be used. Then, using two RCTs, we investigated different methods for measuring outcomes in NPIs for people with early and late stage dementia. We found that there are issues associated with using generic outcome measures for dementia, both in early and late stages of the disease, and that there is scope for developing dementia-specific preference-based measures.

Then, utilising data from Danish national registers, we estimated age- and gender-specific survival times for people with and without dementia, both from prevalent and incident perspectives. We found significant differences in survival between the two groups. This can be used for decision model inputs for future economic evaluations. Propensity score matching was applied to the same data, and used to estimate long-term medical costs in people with and without dementia, and costs attributable to dementia. We found costs increased significantly for the dementia group around the time of diagnosis, and then returning to pre-diagnostic levels for a number of cost categories. These findings provide useful inputs for developing decision models for dementia interventions. Finally, these inputs were used to develop a decision model for economic evaluation of NPIs in dementia, and application of this models demonstrated how a range of pre-diagnostic, diagnostic, and post-diagnostic interventions can be modelled.

Overall, this project demonstrated a need for specialised models for economic evaluation of NPIs in dementia, developed new inputs and provided an example of a model suitable for the task.

Title: $\quad$ Economic issues related to the evaluation and application of telehealth

Candidate: Isabelle Mairey

University: Centre of Health Economic Research (COHERE), Department of Business and Economics, University of Southern Denmark, Denmark

\footnotetext{
Abstract:

The thesis examines the potential of home-based telehealth compared to standard health care in the context of a randomised pragmatic telehealth trial, Ahead In Health in the municipality of Horsens in Denmark. Its novel contributions were: Estimating the cost-utility of telehealth in a multisectorial Danish health care setting; exploring if time preferences affect changes in self-efficacy with telehealth instead of standard care; using an instrumental variable approach in the evaluation of telehealth interventions to assess causal effects while accounting for non-compliance to per protocol.

The intervention involved data transmission and follow-up communication between health professionals and patients diagnosed with diabetes, chronic obstructive pulmonary disease, congestive heart failure, as well as cardiac rehabilitation and geriatric patients. The trial ran for 24
} 
months, starting September 1st 2013, and randomised 149 participants to telehealth and 153 to standard care. Three studies were undertaken using the trial data.

Firstly, an economic evaluation assessed if telehealth in $\mathrm{AiH}$, used as a substitute for standard care, reduced demand for health care services and improved patients' quality of life. A health care sector perspective was adopted. Incremental cost utility analyses compared changes in costs and mean quality adjusted life years (QALYs) gained after 3 and 12 months. No statistically significant differences in mean total costs or QALYs gained after 3 or 12 months were found. The probability of cost-effectiveness was 30\% after 3 and 12 months at an acceptance value in accordance with NICE guidelines of 300,000 DKK $(£ 30,000)$ per QALY gained. Results were sensitive to the exclusion of participants who died during the trial period.

Secondly, we tested the hypothesis that continuous feedback from health personnel under telehealth, compared to standard care, encourages intensified self-monitoring leading to increased self-efficacy in present-oriented patients. Participants completed a survey containing questions on time preferences once and questions on self-efficacy three times during 12 months ( $\mathrm{n}=163)$. Controlling for preferences and baseline characteristics, using a difference-in-difference strategy, no significant incremental changes in self-efficacy were found across trial arms for presentoriented patients.

Lastly, intention-to-treat (ITT) and treatment-on-the-treated (TOT) methods have been applied to deal with non-adherence in telehealth trials. This is problematic due to endogeneity bias. We use an IV-approach to correct this. Treatment assignment serves as a random source of variation in compliance enabling the estimation of the local average causal effect (LATE) on compliers, receiving telehealth instead of standard care, due to their random assignment. Although statistically nonsignificant, QALYs gained were greater than the effect of offering telehealth (ITTanalysis) but less than for those who complied (TOT-analysis). In this intervention, this is equal to the average treatment effect on the untreated, had they been treated. Hence, the results apply to a larger share than just the compliers themselves.

Title: $\quad$ Lifelong physical activity and long-term labor market outcomes

Candidate: Jaana Kari

University: School of Businesss and Economics, University of Jyväskylä, Finland

\begin{abstract}
:
This thesis examines the longitudinal associations between leisure-time physical activity, educational attainment, and labor market outcomes. Data are drawn from the ongoing longitudinal Cardiovascular Risk in Young Finns Study, which is combined with register-based data from Statistics Finland. The thesis consists of four empirical studies organized in separate chapters. The study chapters are preceded by an introductory chapter presenting the background literature and an overview of the thesis, including the research questions, data, and main results.

Chapter 2 analyzes the role of childhood physical activity in academic achievement and subsequent educational attainment. We find that physical activity level and an increase in physical activity level during childhood are positively related to grade point average at the end of compulsory basic education and years of post-compulsory education in adulthood.

Chapter 3 examines the association between childhood physical activity and adulthood earnings. The results show that, among men, childhood physical activity is positively related to long-term earnings calculated over a 10-year period. Among women, no such clear relation is found.
\end{abstract}

Chapter 4 further studies the relationship between childhood physical activity and longterm labor market outcomes by focusing on employment and unemployment. The results indicate 
that childhood physical activity is positively related to employment months and negatively related to unemployment months. As well, persistently active individuals have the highest employment levels and lowest unemployment levels compared with other activity groups.

Chapter 5 scrutinizes the role of annual earnings in self-reported and objectively measured physical activity. The results suggest that higher incomes are associated with higher self-reported physical activity in both genders, whereas the results from the objective measures of physical activity are gender-specific and depend on the measurement day (weekday vs. weekend).

Title: $\quad$ Health and economic aspects of potentially inappropriate medications in older people

Candidate: Virva Hyttinen

University: Department of Health and Social Management, University of Eastern Finland, Finland

\begin{abstract}
:
Health care resources should be used and organised efficiently and equitably in such a way that they produce as much health as possible. This dissertation consists of four sub-studies, whose aims were to determine persons' selection for potentially inappropriate medication (PIM) use, and whether initiation of PIM use is associated with health care service use, costs and mortality in older people.

The data used are from two different population-based cohort studies: data on older people with Alzheimer's disease (AD) between 2005 and 2011, and a 10\% random sample of a general community-dwelling, older population between 2000 and 2013. PIMs were defined by the Meds75+ database maintained by the Finnish Medicines Agency (FIMEA).

People with AD initiated PIM less frequently than those without AD. There were agerelated differences in the factors associated with PIM initiation, e.g. gender, socioeconomic status, in older community-dwelling persons aged $65-74$ and $\geq 75$ years. Overall, PIM initiation was more dependent on patient characteristics, but also on some healthcare system related factors, such as differences in the prescribing of PIM between physicians, and potentially different regional treatment practices.

PIM initiation was statistically significantly associated with hip fractures in people with $\mathrm{AD}$ only after restricting the analyses for the first PIM use period. Also, in the general communitydwelling population, the first PIM use period was particularly associated with an increased risk of fracture-specific hospitalisations and mortality after after considering selection for PIM use. PIM users also had higher hospital costs compared to non-users during the 12-year follow-up.

In conclusion, this dissertation confirms that PIM use is related to a variety of interrelated patient- and physician-level factors. PIM use is associated with an increased risk of negative health outcomes and a greater risk of hospitalisation, and thus, higher hospital costs.
\end{abstract}

Title: $\quad$ The effects of competition and regulation on quality in physiotherapy.

Candidate: Piia Pekola

University: Faculty of Social Sciences and Business Studies, University of Eastern Finland, Finland.

\begin{abstract}
:
In health care, different market mechanisms have become more common in many countries. Competition is believed to increase quality, especially when prices are fixed and patients have a free choice of providers. Intuition dictates that when patients have more choice among providers, demand will be more responsive to quality and firms will only be able to increase revenue by raising quality.
\end{abstract}


Previous literature on quality competition is mainly from the United Kingdom and the United States, and research has focused primarily on hospital markets. The aim of this study was to evaluate the effect com-petition and regulation have on quality in physiotherapy for disabled individuals organised and financed by the Social Insurance Institution of Finland (Kela). Thus, with this thesis it is possible to expand the literature into rehabilitation, such as physiotherapy, which has previously drawn only little attention.

Kela generally uses competitive bidding in its effort to organise physiotherapy. When firms participate in tendering, they define both prices and quality in their tenders, and Kela scores these elements. In addition to organising competitive bidding, Kela has piloted fixed-price service vouchers during the contract period 2011-2014. In this system, where patients had a free choice of providers, only firms able to attract patients turned in revenue. Patient deductibles were not collected.

A previous change in procurement altered the competitive environment of the firms. Thus, the change in the system most likely had an impact on the firms' incentives regarding quality investments. This thesis shows that when quality was measured as firms' quality investments, competition had a negative effect on quality despite the procurement mechanism and the pricing.

Keywords: competition, competitive bidding, free choice, physiotherapy, price regulation, procurement, rehabilitation, service voucher, quality, patient choice

Title: Pharmaceutical expenditures, the reference price system and competition in the pharmaceutical market. A register study

Candidate: Hanna Koskinen

University: Department of Public Health, Faculty of Medicine, University of Helsinki, Finland

\section{Abstract:}

This study examined the impact of the implementation of a generic reference price system on pharmaceutical prices and competition within the market. The focus was particularly on antipsychotic medications. Furthermore, the impact of reference pricing on previously implemented generic substitution was assessed.

Antipsychotics and antidepressants were, in terms of value, among the fastest growing pharmaceutical groups in Finland at the turn of the 21st century. For antipsychotics, most of the cost growth resulted from the rise in the mean daily cost of treatment, whereas the main reason for antidepressant cost growth was the increased number of patients.

The implementation of reference pricing decreased the daily cost of the studied antipsychotics. The decreases ranged from $30 \%$ to $66 \%$ in the short term and from $25 \%$ to $51 \%$ in the medium-to-long term. When the study was extended to other pharmaceutical groups, the average decrease was $35 \%$ at the end of the first year, $56 \%$ at the end of the second year and $60 \%$ at the end of the third year. However, there were large differences in the size of the decrease between groups.

Being included in the reference price system had the largest decreasing impact on prices. However, the reference price system's impact on prices appeared to be waning; the later an active substance was included in the system, the higher the price level remained. In addition, the impact of the reference price system on previously implemented generic substitution remained low, and 2.5 years after the implementation of the reference price system it was almost non-existent.

Generic pharmaceutical markets are highly concentrated in Finland. In addition, there is an overall lack of transparency in the pharmaceutical distribution chain. Further research is needed on the barriers of entry and on the role different operators of the pharmaceutical distribution chain have in promoting price competition in the generic market sector. 
Title: $\quad$ Productivity in the Norwegian hospital sector. Financing, quality, coding and comparability

Candidate: Kjartan Sarheim Anthun

University: Department of Public Health and Nursing, Norwegian University of Science and Technology, Norway

\begin{abstract}
:
In a time of aging populations and technology improvements, hospital services in universal public systems are under increasing pressure. Hospital utilization rates are also relatively greater for elderly, and expectations of medical and technological improvements will continue to increase costs.
\end{abstract}

The aim of this thesis is to identify developments in hospital productivity and its relation to the funding of the hospital sector in Norway during the period 1999 to 2014. The thesis contains four studies: 1) hospital productivity and productivity changes during the period 1999 to 2014, 2) a comparative analysis of hospital productivity growth in Nordic countries, 3) the relationship between hospital quality and productivity, and 4) the relationship between hospital financing and diagnostic coding. The studies are all based on register data from the Norwegian Patient Registry, as well as hospital cost accounting data. Statistical methods suited for large dataset have been utilized.

In study 1 we found that the productivity of Norwegian hospitals had an average weighted growth of $24.6 \%$ from 1999 to 2014, or an annual increase of 1.5\%. The largest gains occurred around the implementation of the hospital ownership reform in 2002. After the hospital reform, most hospitals are larger than what we estimate as optimal size.

Further we compare productivity amongst hospitals in Norway, Sweden, Denmark and Finland. In study 2 we estimated the mean productivity in Norway to be 56.6 per cent compared to the best Nordic hospitals, all being Finnish. If we compare productivity only within each country, the (technical) efficiency in Norway is as high as in Finland. A possible explanation of productivity differences is quality differences. In study 3 we found large differences between hospitals regarding both readmission rates and mortality. Norway had higher readmission rates than the other countries, but the lowest mortality rates. No clear cost-quality trade-off pattern was revealed.

An alternative explanation of productivity growth is that hospitals have changed diagnostic coding practices. This would exaggerate the measurable productivity growth over time. Study 4 show that there is an association between price incentive and the use of complicated diagnoses. However, the effect of price changes is smaller.

Title: $\quad$ Economic evaluation of malaria prevention in Ethiopia: economic burden, equity, and cost-effectiveness analysis of malaria prevention in south-central Ethiopia.

Candidate: Alemayehu Hailu

University: Centre for International Health / Global Health Priorities, Department of Global Public Health and Primary Care, University of Bergen, Norway. School of Public Health, Addis Ababa University, Ethiopia.

\begin{abstract}
Background: Remarkable progress has been achieved in the global effort against malaria in the last decade. Approximately seventy percent of the achievement can be immediately assigned to "whole" anti-malaria interventions, in particular long-lasting insecticidal nets (LLIN) and indoor residual spraying (IRS). Yet the disease continues to be a huge challenge to the health systems of many African countries, and the existing evidence about the economics aspect of malaria
\end{abstract}


prevention is scanty. In addition, little is known about the status of the bed-nets and indoor residual spraying across different layers of wealth status.

Objectives: The aims of this thesis were to estimate the economic burden of malaria; to evaluate the cost-effectiveness of a combined implementation malaria prevention interventions (LLINs and IRS); and analyse the distributional (equity) implications of the interventions southcentral Ethiopia.

Methods: Studies included in this thesis were conducted from 2014 - 2016 in the Adami Tullu district of Oromia Region, in Ethiopia. We conducted a cost of illness using 190 malaria patients in the first study. In Paper II, we carried out a combination of trial-based and literaturebased cost-effectiveness analysis using Markov modelling. In the third study, using a crosssectional data from 6069 households we did an inequality analysis of ownership of LLINs and IRS status. The principal component analysis technique was used for ranking households based on socioeconomic position. We measured the inequality in LLINs and IRS using concentration indices and concentration curve (Paper I\&III).

Results: The median cost of malaria per episode to the household was USD 5.06 (IQR: $2.98-8.10$ ) and the direct cost was significantly higher among the poor. The trial-based analysis had shown that the routine practice dominates both the combined intervention and singleton intervention while the literature-based analysis had indicated that combined intervention had an Incremental Cost-Effectiveness Ratio of USD 1403 per DALY averted. Immediately before we started the trial, the LLIN ownership was $11.6 \%$ and IRS coverage was $72.5 \%$. We found a concentration index of 0.0627 for LLINs and - 0.0383 for the IRS. Inequality in LLIN ownership was mainly associated with a variability in a housing situation, the size of the household, and access to mass media and telecommunication service.

Conclusions: The economic burden of malaria to the rural households in Ethiopia is huge-mainly to the poor. Based on the trial-based cost-effectiveness analysis, we conclude that the combination of LLINs and IRS is not likely to be a cost-effective option compared with singleton intervention. However, based on the literature-based analysis, the combined intervention had potential to be a cost-effective alternative at 3 times GDP per capita per DALY averted. Furthermore, the ownership of LLIN was very low and significantly pro-rich, while IRS status was equitable across socioeconomic strata.

Title: $\quad$ The Health Benefits, Resource Use and Cost-Effectiveness of Current and Future Cervical Cancer Screening Policies in Norway

Candidate: Kine Pedersen

University: Department of Health Management and Health Economics, University of Oslo, Norway

\begin{abstract}
:
In Norway, cervical cancer is the third most common cancer among mid-adult women who are in their productive social and working years. This is despite substantial reductions in cervical cancer incidence following more than two decades of organized cytology-based screening. The discovery of human papillomavirus (HPV) as a carcinogen has led to new technologies that are changing the landscape of cervical cancer prevention, including HPV-based screening approaches and HPV vaccination. The thesis addresses knowledge gaps related to the value of implementing these technologies in clinical practice, and whether to adapt the screening program for women who were vaccinated against HPV infections in adolescence. To evaluate candidate strategies for current and future cervical cancer screening policies, the thesis includes four decision-analytic studies that employ mathematical simulation modeling. Each study identifies attractive strategies that improve
\end{abstract}


the cost-effectiveness and help balance the health benefits and resource use associated with these policies.

Specifically, for the current cytology-based screening program, Paper I indicates that the efficiency and effectiveness (in terms of precancer detection) can be improved using reflex HPV mRNA testing to triage women with minor cervical cytological lesions. However, the long-term consequences of this strategy should be demonstrated prior to implementation. In order to improve the long-term efficiency and effectiveness (in terms of cervical cancer incidence), Paper II suggests to triage women with minor cervical cytological lesions using reflex HPV DNA testing with direct colposcopy referral for women positive for HPV-16/-18 infections. For the future cervical cancer screening policies in Norway, Paper III indicates that in order to maximize the cervical cancer preventive benefits of primary HPV-based screening while controlling colposcopy referral rates, HPV-based screening should start at an earlier age (i.e., <34 years) and rather utilize a less intensive triage algorithm for HPV-positive/cytology-negative women. Finally, in order for screening to remain cost-effective for women who received the HPV vaccine in adolescence, Paper IV suggests that a de-intensified HPV-based screening strategy (e.g., screening once or twice over a lifetime) is required. These findings provide knowledge to help decision-makers in their continuous and complex work to refine cervical cancer prevention policies in Norway.

Title: $\quad$ Sickness absence and disability benefits: The importance of workplaces, attitudes and nurture

Candidate: Marte E. S. Ulvestad

University: Frisch Centre and Department of Economics, University of Oslo, Norway

\begin{abstract}
:
What factors are important for the understanding of sickness absence and disability benefit receipt? Knowing the answer to this question is essential to avoid unnecessary use of these benefits. This is important as sickness absence and disability insurance participation are costly to society in many respects: public expenditures, high expenses for businesses, represent lost productivity, and exclusion from the labour market might be harmful for the individual, both socially and financially.

The first chapter of the thesis studies disparities in sickness absence between workplaces and asks to what extent these disparities are related to characteristics of the workplace itself. To answer this question, detailed administrative register data comprising the Norwegian labour market between 1993 and 2011 are employed. Using a two-way fixed effect model, the importance of the workplace is disentangled from the importance of unobservable employee characteristics, and their relative importance for disparities in sickness absence levels between workplaces is measured. The analysis reveals that about 40 percent of the sickness absence differences can be attributed to fixed characteristics of the workplace itself, while the remaining 60 percent is attributed to unobservable employee heterogeneity.

The second chapter examines the relationship between attitudes and sickness absence, analysing whether attitudes can explain parts of the variation in sickness absence associated with gender, age, education and immigration status. For this study, data have been collected from two different sources. A survey, aimed at identifying attitudes, has been conducted among employees within public health care. In addition, detailed data on sickness absence for the respondents were assembled from the employer. By linking these two data sources, we obtain information on the actual absence behaviour of the employees together with subjective assessments. The analysis reveals differences in attitudes between demographic groups that also differ in their average sickness absence rates, but there is no evidence of attitudes being able to explain these differences.
\end{abstract}


The last chapter concerns the role of nature and nurture for the intergenerational transmission of labour market outcomes, including sickness absence and disability benefits. A sample of adoptees from South Korea, effectively randomly assigned to their adoptive parents, is studied to examine the importance of nurture, environmental factors, for the intergenerational transmission. To disentangle the importance of nurture and nature, the sample of adoptees is also compared to non-adopted children. The analysis shows that the intergenerational transmission of sickness absence and disability benefits is small and non-significant. Furthermore, the results indicate that heritability accounts for about 70 percent of the correlation across generations, across the labour market outcomes studied.

Title: $\quad$ Distributional effects of payment for performance in the health sector: Examining effects on structural quality, performance outcomes and service utilisation in Tanzania

Candidate: Peter John Binyaruka

University: Department of Public Global Health and Primary Healthcare, University of Bergen, Norway

\begin{abstract}
:
Introduction: Most evaluations of $\mathrm{P} 4 \mathrm{P}$ have focused on average programme effects on the incentivised services, paying little attention to distributional effects of payment-for-performance (P4P). Specifically, little is known about the effects of $\mathrm{P} 4 \mathrm{P}$ on structural quality of care (e.g. availability of medical commodities), and similarly on the understanding of the heterogeneity of the P4P effects across subgroups of providers and populations. This PhD work fills that knowledge gap using data from Tanzania.

Data sources: The study collected data in intervention and control areas through facility and household surveys, and facility payments data from administrative records. Baseline data were collected in January 2012 with a follow-up 13 months later. Facility survey across 150 facilities included data on the availability and stock-out of medical commodities and facility characteristics. Household survey across 3000 households (20 per facility catchment area), and a similar sample size in the follow-up survey, captured information about individual and household characteristics and service utilisation.

Analyses: A difference-in-differences (DID) regression model was used to estimate the average effects of P4P on the availability and stock-out of medical commodities (Paper I) and differential effects of P4P across facilities' subgroups (Paper I and II), and across populations subgroups (Paper III). Descriptive measures of inequality were also used to assess the distribution of facility payouts across facilities' subgroups (Paper II).

Results: Paper I reports that P4P improved the availability of drugs and supplies and reduced their stock-out rates, but had no effect on the availability of medical equipment. The improved effects were generally similar across facilities, but relatively higher among facilities serving a poor population and located in rural areas. Paper II finds that facility payments were initially higher among higher level facilities (hospitals and health centres than dispensaries), the better resourced than worse resourced facilities, and facilities serving wealthier than poorer populations, but these inequalities in payouts declined over time. The effect of P4P on institutional delivery rates was greater among facilities with low baseline performance, serving middle wealth populations, located in rural areas, than among their counterparts; whereas the effect on provision of antimalarial drugs was similar across facilities subgroups. Paper III finds that the effect of P4P on institutional deliveries was greater among women in the poorest households, who lived in rural areas and who did not have health insurance than among their counterparts. P4P effect on the uptake of antimalarial drugs was equally distributed across population subgroups.
\end{abstract}


Conclusion: P4P programme can improve structural quality of care in terms of the availability of medical commodities. It can further enhance more equitable performance among facilities as the worse-off providers improved most in this study. Similarly, P4P can enhance equitable service utilisation since the service use increased mostly among the worse-off populations.

Title: Health and Unemployment in Norway: Selection or Causation?

Candidate: Silje L. Kaspersen

University: Department of Public Health and Nursing, Norwegian University of Science and Technology (NTNU), Norway

\begin{abstract}
:
Aims: In this research project we aimed to; 1) study the association between ill health and future unemployment (health selection), 2) detect the causal effects of exposure to organisational downsizing on employees' mental and physical health, measured as changes in prescribed drug purchases, and 3) detect the timing of initiation of psychotropic medication in relation to unemployment in the months before, during, and after job loss, to detect the period of greatest risk. Potential effect measure modification was explored by sex, age, and education.

Methods: Health selection was explored in a prospective cohort study based on adults ( $\geq$ 20 years) who participated in HUNT2 (1995-1997), linked to administrative registers from 1995 to $2008(\mathrm{~N}=36,249)$. Cox proportional hazard models were used to estimate hazard ratios of timeto-registered unemployment. The effect of job insecurity on health was investigated in a quasinatural experiment on the Norwegian working population by using individual-level panel data. We studied changes in prescribed drug purchases for those exposed to major organisational downsizing in the period 2004-2012 $(\mathrm{N}=144,089)$. A random effects logistic regression estimator was used to estimate odds ratios with 95\% confidence intervals. Finally, the timing of initiation of antidepressants $(\mathrm{N}=34,111)$, hypnotics and/or sedatives $(\mathrm{N}=32,570)$, anxiolytics $(\mathrm{N}=26,838)$, and antipsychotics $(\mathrm{N}=12,495)$ in relation to unemployment in the months before, during, and after job loss was investigated by using a case-crossover design for the period 2005-2010. A conditional fixed-effects estimator and odds ratios with 95\% confidence intervals were used to measure the relative risk of being unemployed at the time of drug purchase (case period) compared with being in the same state of unemployment 12, 24, and 36 months (control periods) before the drug purchase took place.

Results: Those with high baseline (1995-1997) symptom levels of anxiety and depression or a number of chronic somatic conditions had almost twice the risk of becoming unemployed in the period 1995-2008 compared with their healthier peers. We found substantial negative effects of downsizing on both mental and somatic health, and a twofold to threefold increase in the risk of first-time purchase of psychotropic drugs during the month before the date of unemployment, with an increasing trend in the three months ahead of unemployment. In the six months after the end of unemployment, the odds ratios were close to those of six months before job loss. The same trends were present for drugs prescribed for somatic and pain conditions, but with substantially lower risk estimates than for psychotropics.

Conclusions: We found evidence of both health selection effects into unemployment and negative effects of downsizing and unemployment on health in the Norwegian working population.
\end{abstract}


Title: $\quad$ Economic analysis of Results-based financing in Malawi. Strengthening the evidence base for alternative maternal and perinatal Healthcare funding

Candidate: Jobiba Chinkhumba

University: Centre for International Health, Department of Global Public Health and Primary

Care, University of Bergen, Norway. Department of Community Health and Family Medicine, University of Malawi, College of Medicine, Malawi. Institute of Public Health, University of Heidelberg, Germany.

\section{Abstract:}

Encouraging women to deliver their babies in health facilities is one way of increasing coverage of skilled care at the time of delivery. Malawi embarked on an initiative called Results-based financing (RBF) which provides conditional financial incentives to health workers to increase quality delivery care and to mothers for delivering in health facilities. Yet, the benefits of facility compared to homebased births; the impact of RBF on obstetric emergency care seeking and associated costs, and the evidence for RBF efficiencies are lacking.

I conducted three studies. I examined maternal and perinatal benefits of facility-based births relative to home births through a literature review and meta- analysis of population-based cohort studies reporting on maternal and perinatal outcomes by place of delivery in sub-Saharan Africa. A total of 36,772 pregnancy episodes were included in the analyses. Relative to facility births, perinatal mortality was higher among home births, but the difference was only significant when produced with a fixed effects model (OR 1.21, 95\% CI: 1.02-1.46) and not when produced by a random effects model (OR 1.21, 95\% CI: 0.79-1.84). There was increased risk of maternal mortality for facilitybased relative to home deliveries (OR 2.29, 95\% CI: 1.58-3.31).

Using a pre-and post-design with controls, I used generalised linear models to ascertain the impact RBF had on time to seek care for women experiencing pregnancy related complications and associated household costs. I used primary household survey data collected at baseline in 2013 and repeated in 2014 (midline) and 2015 (endline), involving a total of 2, 219 women experiencing complications in their most recent pregnancy before the surveys. Receipt of RBF was associated with reduced expected mean time to presentation for facility care for women experiencing complications. Relative to non-RBF areas, time to seek care in RBF areas decreased by $27.3 \%$ (95\% CI: 28.4-25.9) at midline and 34.2\% (95\% CI: 37.8-30.4) at endline. There was no demonstrable effect of RBF on overall (direct + indirect) household costs.

Thirdly, I used a decision tree model to estimate expected RBF efficiency in terms of cost per deaths averted and life years gained. Relative to the status quo, RBF cost $\$ 42.83$ per additional birth, averted 0.0015 death and gained 0.0410 life year. Thus, RBF had an incremental cost-effectiveness ratio per death averted and life year gained of \$29,135 and \$1,045, respectively. At a willingness to pay of $\$ 1,446$ ( 3 times Malawi Gloss Domestic Product per capita), RBF had $60 \%$ probability of being cost-effective.

Policies that promote facility-based births can increase perinatal survival, but their benefits on maternal health would depend on concomitant quality improvement efforts. RBF encourages women with pregnancy related complication to present for facility care early which may lead to better outcomes. RBF appears borderline cost-effective at high levels of willingness to pay and unlikely to be cost-effective at less than 3 times GDP per capita.

Title: $\quad$ Universal Health Care Supply: Queues, Prices and Regional Variation

Candidate: Ingrid Huitfeldt

University: Frisch Centre and Department of Economics, University of Oslo, Norway

\footnotetext{
Abstract:

The thesis consists of four papers related to Norwegian healthcare system.
} 
Paper 1: Spending the Night? Provider Incentives, Capacity Constraints and Patient Outcomes. This paper studies the effects of a change in the payment scheme for hospitals in Norway. In 2010, payments for stays lasting longer than one day were substantially increased, while payments for patients discharged on the day of admission were decreased. This gave hospitals incentives to shift patients from one-day stays to two-day stays, or to decrease the volume of one-day stays. I study hospital responses by exploiting the variable size of price changes across diagnoses in a difference-in-differences framework, and account for marginal costs of overnight stays through a measure of time-varying capacity constraints. I find no evidence that hospitals respond to price changes.

Paper 2: Waiting for Surgery: Effects on Health and Labor Supply (co-authors: Anna Godøy, Venke Haaland and Mark Votruba). In universal health care systems, patients often face significant wait times for treatment when capacity constraints are binding. We estimate the effects of wait time for orthopedic surgery (days from referral to surgery) on health and labor market outcomes, using microdata covering all publicly funded orthopedic surgeries in Norway referred in 2010 and 2011. Our identification strategy exploits quasi-random variation in wait times generated by system congestion at the time of patients' entry into the queue. We find that longer wait times for surgery significantly increase health related work absence. There does not appear to be any lasting health implications.

Paper 3: Regional Variation in Health Care - Does Education Matter? (co-author: Anna Godøy). Geographic variation in healthcare utilization has raised concerns of possible inefficiencies in healthcare supply in high utilization regions, as these regions typically do not achieve better health outcomes. However, geographic variation in utilization could also reflect variation in the demand for healthcare, arising from patient sorting. We separate healthcare utilization between place and patient by exploiting migration across hospital regions. Using detailed patient data covering the entire Norwegian population during 2008-2013, we find that place-specific factors account for roughly $40-55 \%$ of the total variation in hospital utilization, while the rest is explained by patient characteristics. We further document heterogeneous impacts of hospital regions across socioeconomic groups: place factors account for $60-90 \%$ of variation in utilization for high school dropouts, compared to $20-45 \%$ for patients with a college degree.

Paper 4. Universal Breast Cancer Screening and Mortality. This paper studies effects of universal breast cancer screening with mammography on all-cause mortality. The staggered implementation of the Norwegian mammography program provides spatial and temporal variation in invitation to screening. Results indicate that mammography screening has no significant effect on all-cause mortality. The confidence intervals are not small enough to reject effect sizes found in medical studies on breast cancer mortality.

Title: $\quad$ The Impact of Financial Incentives on Demand and Supply of Health Care Services in Norway: Empirical Studies on Co-Payments and Activity-Based Hospital Financing

Candidate: Camilla Beck Olsen

University: Department of Health management and health economics, Institute for Health and Society, University of Oslo, Norway

\footnotetext{
Abstract:

The financing of health care systems is constantly evolving in order to best achieve objectives such as access to and efficient use of resources. To be able to use financial incentives efficiently, there is need for evidence on to what extent users and providers respond to these incentives. The aim of this thesis is to provide more evidence across different populations, settings and outcomes
} 
on how behaviour in health care systems is affected by financial incentives. Paper I estimates the effect of a co-payment exemption on adolescent visits to the General Practitioner (GP). Paper II tests to what extent hospitals focus on the diagnostic groups that are most financially favourable given costs and reimbursement rates, while, Paper III evaluates to what extent a $10 \%$ price increase was successful in inducing hospitals to provide more day surgery.

Overall, the findings in this thesis support the existing empirical literature that financial incentives do affect behaviour. The results suggest that adolescents were sensitive to having to pay a fee for visiting their primary care physician and that exempting them from this fee increased their use of health care services by $22.1 \%$ among females and $13.8 \%$ among males. Hospitals also respond to changes in reimbursement for Diagnostic Related Groups (DRGs). The percentage increase in number of admissions was on average four times higher when the DRG reimbursement was increased, relative to the percentage increase in DRGs with reduced reimbursement rates. However, a $10 \%$ increase in DRG weight for day surgery did not seem to provide a strong enough incentive for hospitals to provide more day surgery. The results of the analyses are relevant for both policy-makers and researchers who are interested in the further development of both demandside and supply-side incentives to achieve health policy objectives such as access and efficiency.

Title: $\quad$ Caring, Sharing, and Childbearing: Essays on Labor Supply, Infant Health, and Family Policies

Candidate: Anna Norén

University: Department of Economics, Uppsala University, Sweden

\begin{abstract}
:
In Essay 1, I study the consequences on labor market outcomes and sick leave of having an elderly parent in need of care. Using Swedish register data I compare the labor market outcome trajectories of adult children before and after their parent suffer a health shock. I find that employment and income of adult children are slightly reduced in the years leading up to the demise of their parent, but that the size of the impact is largest in the year, and the year after, parental demise. No effects on labor market outcomes are found from having a parent suffering stroke. Taken together, the results suggest that the opportunity costs of parental care need in the form of adverse labor market impacts are small.

In Essay 2 (co-authored with Erik Grönqvist, Anna Sjögren and Helena Svaleryd) we exploit the stepwise introduction of alcohol screening and brief interventions at Swedish antenatal clinics, to evaluate the causal effect of enhanced alcohol prevention on infant health using a difference-in-differences strategy. The detrimental effects of alcohol exposure in utero are well documented, and therefore identifying effective methods for preventing harmful maternal alcohol consumption is of great importance. We find that the program improves infant health measured by prescription of pharmaceutical drugs and hospitalizations during the child's first year of life. The results suggest that effects are likely driven by changes in maternal behavior after the first trimester and seem to extend beyond the birth of the child.

Essay 3 (co-authored with Erik Grönqvist, Anna Sjögren and Helena Svaleryd) examines the effects of targeted preventive interventions for pregnant women with elevated alcohol risk on infant health and maternal behavior. We exploit the discrete nature in the decision rule to provide an alcohol preventive intervention to mothers at risk in a regression discontinuity design. The results suggest that the intervention has negligible impact on birth weight and small effects on the gestational age. We are unable to determine if this is due to a low effectiveness of the intervention or due to a low take up of the intervention.
\end{abstract}


In Essay 4, I investigate whether a reduction in the care burden, or a decreased nonmonetary cost, of parental leave through the availability of childcare for older siblings affects how the leave is divided between mothers and fathers. The effect of access to childcare is evaluated by utilizing the regional heterogeneity of the implementation of a childcare reform in Sweden in 2002 that gave children of parents on parental leave with a younger sibling the right to stay in childcare. Results suggest that availability of childcare for an older sibling during parental leave does not impact the division of parental leave between mothers and fathers.

Title: An assessment of renal replacement therapy: A register-based study on equality of access, cost, effectiveness, and cost-effectiveness of kidney transplantation in Sweden

Candidate: Ye Zhang

University: Faculty of Medicine, Lund University, Sweden

\begin{abstract}
:
Renal replacement therapy, which includes dialysis and kidney transplantation, is a lifesaving treatment for patients with end-stage renal disease. Kidney transplantation is generally preferred over dialysis as it is associated with lower mortality, higher quality of life, and lower costs. Despite these advantages, the availability of kidney transplant to all who can benefit from it is limited by the scarcity of kidneys. The overall purpose of this thesis was to explore equality of access to kidney transplantation as well as the effectiveness, cost, and cost-effectiveness of kidney transplantation relative to dialysis.

The analyses were performed using data from the Swedish Renal Register, a Swedish national register for renal replacement therapy patients, which was linked to other national registers and regional healthcare utilization databases. Logistic regression models and Cox regression models were applied to study the association between income and education and access to kidney transplantation in general, access to the waitlist, and access to kidney transplantation conditional on waitlist placement. The double robust inverse-probability-weighted regression adjustment approach was applied to estimate the potential outcome mean and the average treatment effect on survival time and healthcare costs.

The results showed that patients with higher income and education had a higher chance of access to kidney transplantation in general than patients with lower income and education. After dividing the kidney transplantation process into access to the waitlist and access to kidney transplantation conditional on waitlist placement, the chance of waitlist access was larger than that of kidney transplantation access conditional on waitlist placement for both higher income and education patients. The survival advantage of kidney transplantation compared with dialysis was around 14 years. There was no significant difference in the survival advantage of kidney transplantation between men and women. Kidney transplantation was not cost saving in the first year after kidney transplantation, but became cost-saving for the second year, the third year, over the three years, and over lifetime after kidney transplantation.

In conclusion, the findings of this thesis show that there are inequalities related to socioeconomic status in the process of kidney transplantation. Moreover, the inequality in access to the waitlist is substantially larger than the inequality in access to kidney transplantation conditional on waitlist placement, and is therefore expected to contribute more to socioeconomicstatus-related inequalities. Kidney transplantation increases survival time and reduces healthcare costs relative to dialysis treatment. This combination of inequality in access and advantage of transplantation over dialysis means that patients with advantageous socioeconomic status will have
\end{abstract}


longer survival time and lower healthcare costs. However, there is no sex inequality in either access to kidney transplantation or survival advantage of kidney transplantation.

Title: $\quad$ Health, inequality and the impact of public policy

Candidate: Gawain Heckley

University: Faculty of Medicine, Lund University, Sweden

\section{Abstract:}

This thesis combines three large areas of economic research: the measurement of socioeconomic related health inequality; the decomposition of socioeconomic related inequality into its explanatory factors; and the treatment effects and policy evaluation literature. The thesis is comprised of four papers that stand as individual contributions but together they have a common theme: they assess the impact of public policy (education and drinking age laws) on health and health inequality.

Overall, using three different quasi-experiments to identify exogenous variation in education, no clear evidence is found for a health improving impact of education on health. No impact of education on health is found using variation in education identified by either a twins differencing strategy or variation in schooling induced by two major compulsory school reforms. Using eligibility rules for university education combined with RD, evidence is found that university education leads to greater contraceptive use amongst women, but this impact may just be a consequence of family planning rather than a health investment per se.

The same quasi-experimental techniques are applied in combination with the new decomposition method developed as part of this thesis to assess whether differences in education can explain income related health inequality. Even though a clear association between education levels and income related inequality in health is observed, no clear impacts of education on income related inequality are observed once a more convincing control strategy is used.

Sweden's Minimum Legal Drinking Age (MLDA), however, does appear to have an impact on alcohol consumption and in particular episodic heavy drinking. The combination of the specific design of Sweden's two part MLDA and other alcohol control policies in place appear to both protect young underage adults and also mitigate the large negative health effects of increased alcohol consumption in the transition to full legal access to alcohol. In particular the large increases in mortality that accompany the large increases in drinking observed in the United States at age 21 when purchasing of alcohol is legalised are not observed in Sweden even though large increases in drinking in Sweden are observed.

Thesis Title: Health economic evaluation for evidence-informed decisions in low-resource settings. The case of Antenatal care policy in Rwanda.

Candidate: Regis Hitimana

University: Public Health and Clinical Medicine (Epidemiology and Global Health unit), Umeå University, Sweden

\footnotetext{
Abstract:

Introduction: This thesis aims to contribute to the use of health economic evidence for informed health care decisions in low-resource settings, using antenatal care (ANC) policy in Rwanda as a case. Despite impressive and sustained progress over the last 15 years, Rwanda's maternal mortality ratio is still among the highest in the world. Persistent gaps in health care during
} 
pregnancy make ANC a good candidate intervention that can, if improved, contribute to better health and well-being of mothers and newborns in Rwanda.

Methods: The primary data sources were a cross-sectional household survey ( $\mathrm{N}=922)$, a health facility survey $(\mathrm{N}=6)$, and expert elicitation with Rwandan specialists $(\mathrm{N}=8)$. Health-related quality of life (HRQoL) for women during the first-year post-partum was measured using the EQ5D-3L instrument, and associations between HRQoL, adequate ANC utilization according to the Rwandan guidelines, and socioeconomic and demographic predictors were tested (Paper I). The societal cost of current ANC practices in Rwanda was estimated (Paper II). Incremental cost and health outcomes of implementing the 2016 World Health Organization (WHO) ANC recommendations compared to current practice in Rwanda were estimated (Paper III). The last study was a systematic review of evidence published between January 1999 and April 2018 on the cost and cost-effectiveness of routine ultrasound during pregnancy (Paper IV).

Results: Most (61\%) women had not adequately attended ANC during their last pregnancy; either attending late, or fewer than four times. Adequate ANC utilization, good social support, and household wealth, were significantly associated with better HRQoL measured using EQ-VAS. The most prevalent health problems were anxiety or depression and pain or discomfort. The first ANC visit accounted for about half the societal cost, which was \$44 per woman (2015 USD) in public/faith-based facilities and \$160 in the surveyed private facility. Implementing the 2016 WHO recommendations in Rwanda would cost between $\$ 5.8-\$ 11 \mathrm{~m}$ annually across different attendance scenarios. Perinatal mortality would reduce by $22.5 \%-55 \%$ and maternal mortality by 7\%-52.5\%. Four out of six combinations of attendance and health outcome scenarios were below the GDP-based cost-effectiveness threshold. Only nine studies on cost and cost- effectiveness of ultrasound during pregnancy reached the data extraction stage. Routine ultrasound screening was reported to be a cost-effective intervention for screening pregnant women for cervical length, vasa previa, and congenital heart disease, and cost-saving for screening for fetal malformations.

Conclusions: The use of health economic evidence in decision making for low-income countries should be promoted. It is currently among the least used types of evidence, yet there is a huge potential of gaining many QALYs given persistent and avoidable morbidity and mortality. Low-income countries, particularly those that that still have a high burden of maternal and perinatal mortality should consider implementing the 2016 WHO ANC recommendations.

Title: $\quad$ Essay on Malnutrition, Savings and Preferences

Candidate: Tharshini Thangavelu

University: Department of Business Administration, Technology and Social Sciences, Luleå University of Technology, Sweden

\footnotetext{
Abstract:

The thesis address questions about the economic well-being of heterogeneous households and individuals and how they cope with scarcity and constraints in the light of the three themes, namely, malnutrition, savings and preferences. The first two papers are self-written and deals with child malnutrition in India.

In the first paper, I quantify the impact of malnutrition on psychosocial abilities (aspiration, self-efficacy and self-esteem) using an instrumental variable approach. I do this by employing Young Lives panel data from Andhra Pradesh in India and self-reported drought variables as instruments to isolate the causal path, the results suggest that malnutrition leads to reduced self-efficacy whereas aspiration and self-esteem remain unaffected by malnutrition. Further analysis reveals that boys' self-efficacy are negatively affected by malnutrition, while the
} 
results for girls have no statistical power. These results contributes to the literature, which has so far mostly focused on the consequences of malnutrition on children's cognitive skills.

The second paper takes on a quantile regression to compare with least square estimation, which is typically used in malnutrition research to determine the average relationship of particular household characteristics on a child's height-for-age z-score (HAZ). I use of two birth cohorts of children of the same age in the Indian state of Andhra Pradesh. Although the constant catch-up effect along the distribution of HAZ could be rejected, the lower end of the quantile indicated a partial catch-up effect in children's past nutritional deficiency. The results suggest no improvement in malnutrition recovery across the two birth cohorts.

The third paper tries to understand farmers saving behaviors in Malawi. The paper replicate and reanalyze data from the randomized controlled trial of a programme originally carried out by Brune et. al (2016) to facilitate formal saving for Malawian tobacco farmers. The results from various estimation analyses that were carried out also broadly support the conclusions from the original study. We also conducted a separate analysis focusing on the subset of farmers who chose to make use of the savings vehicles offered. Our findings were that, compared with the overall treatment group, this subset of farmers had far greater positive effects on their agricultural output.

While, in the last paper, my coauthors and I set-up a stated preference methods to study anglers willingness to pay for improvements in the characteristics of fishing sites in the county of Jämtland in Sweden. We use two existing angling sites, and hypothetical sites similar to these, to explore transferability of responses between different sites and to examine the welfare effects of improvements in fishing site characteristics. We find that anglers have highly heterogeneous preferences, and that modelling this heterogeneity using latent class models leads to different classes being estimated for the two different sites studied. 